\title{
YIELD CURVE DYNAMICS: REGIONAL COMMON FACTOR MODEL
}

\author{
Boril Šopov, Jakub Seidler*
}

\begin{abstract}
:
In this paper, we focus on thorough yield curve modelling. We build on extended classical NelsonSiegel model, which we further develop to accommodate unobserved regional common factors. We centre our discussion on Central European currencies' yield curves: CZK, HUF, PLN and SKK. We propose a model to capture regional dynamics purely based on state space formulation. The contribution of this paper is twofold: we examine regional yield curve dynamics and we quantify regional interdependencies amongst considered currencies' yield curves. We conclude that the CZK yield curve possesses its own dynamics corresponding to country specific features, whereas other currencies' yield curves are strongly influenced by the regional level, the regional slope factor or both.
\end{abstract}

Keywords: dynamic factor model, Kalman filter, Nelson-Siegel, state space, regional yield curve, principal component analysis

JEL Classification: C51, C53, G17

\section{Introduction}

Yield curve dynamics heavily affects financial institutions' decision-making thus good understanding of underlying driving forces is essential. Also, importance of a proper yield's curve modelling has been increasing since marking-to-market practice has become a common standard. Moreover, the shape of a yield curve can give valuable information about the expected inflation and overall economic activity.

As financial integration proceeds we can expect the importance of country specific dynamics to gradually diminish. Therefore, we need a framework capable of modelling several yield curves of different countries at once. With such a framework, we are able to analyse dynamics and possible structural breaks at regional level and identify common or country specific factors influencing particular yield curves.

We mostly build on works of Diebold et al. (2006) and Diebold et al. (2008) that introduced a dynamised version of classical Nelson-Siegel model and discovered a

* Boril Šopov, Institute of Economic Studies, Charles University in Prague (boril.sopov@ gmail.com); Jakub Seidler, Czech National Bank and Institute of Economic Studies, Charles University in Prague (seidler@email.cz). Authors thank anonymous referee for useful suggestions. Both authors acknowledge the support by the Czech Ministry of Education (Grant MSMT 0021620841) and SVV 261 501. Jakub Seidler also acknowledges the support by the Grant Agency of the Czech Republic (GACR 403/10/1235).

The findings, interpretations and conclusions expressed in this paper are entirely those of the authors and do not represent the views of any of the above-mentioned institutions 
global yield curve. We proceed notionally in a similar fashion, extend the model and endeavour to assess existence of regional yield curve for selected countries in Central European region - the Czech Republic, Slovakia, Poland and Hungary.

We confirmed the existence of a regional yield curve and further examine how much each currency yield curve is affected by common regional factors.

The paper is organized as follows: Section 2 summarises related literature regarding the yield curve modelling. Following part introduces Regional Common Factor Model embedded in the state space framework. Section 4 presents the estimation technique and model's results. The paper is concluded in the last section.

\section{Related Literature}

Nelson and Siegel (1987) published a seminal paper in 1987 on modelling yield curve dynamics. This paper introduced a parsimonious way to model the whole yield curve with only three parameters. This approach allowed to reproduce all stylised facts about possible yield curve shape: monotonic, humped or S-shaped (Nelson and Siegel, 1987, p. 474).

Further developments were introduced in Litterman and Scheinkman (1991). The main novelty was the identification of three main factors explaining up to $97 \%$ of variance of returns. These three factors were called: level, steepness (slope) and curvature.

Diebold et al. (2006) introduces the Nelson-Siegel model into state space framework thus allowing for clear inference and one step estimation. Additionally, they illustrated how the forecasting results can be further improved, if macroeconomic variables as regressors are included into the state space framework.

Last but not least, Diebold et al. (2008), dealing with Nelson-Siegel yield curve modelling from statistical perspective, proposed an idea and confirmed the existence of global yield curve, which affects country specific yield curve.

In this paper, we further develop the above mentioned concept of the global yield curve focusing our attention to selected Central European countries with effort to identify their common regional yield curve.

As regards yield curves of individual countries of our interest, Málek et. al (2007) confirmed the Nelson-Siegel yield curve approach to be appropriate for the Czech yield curve smoothing. A growing utilization of this framework is represented also by Reppa (2009), who used the dynamised Nelson-Siegel approach to model the Hungarian yield curve dynamics with use of macroeconomic variables. The zloty yield curve modelling is further reviewed by Marciniak (2006).

Although yield curve dynamics is a well-studied topic, to our best knowledge, our study is novel in terms of application of the proposed method to extract regional latent factors in selected countries of Central Europe. There are several studies that deal with cross country yield-curve dynamics based on dynamised Nelson-Siegel framework (e.g. Mehl, 2009 or Hoffmaister et. al, 2010), however, this paper takes a different approach and models regional common dynamics for all considered countries together. 


\subsection{Classical Nelson-Siegel Model}

As it was stated, Nelson and Siegel (1987) proposed static model based on three exponential components. Having parsimony in mind, they put forward a model with only $3+1$ parameters to capture the entire yield curve. ${ }^{1}$

We use the specification given in Diebold et al. (2006), who restate the classical Nelson-Siegel model as in equation (1). Moreover, as Nelson and Siegel (1987, p. 475) points out, if we assume that spot rates are generated by a differential equation, then the forward rates are given as a solution to a second-order differential equation with real and unequal roots (Nelson and Siegel, 1987, p. 475), which gives a straightforward inference of the model. That is why the Nelson-Siegel model relies on exponential components that take different values for various maturities.

$$
y(\tau)=l+s\left(\frac{1-e^{-\lambda \tau}}{\lambda \tau}\right)+c\left(\frac{1-e^{-\lambda \tau}}{\lambda \tau}-e^{-\lambda \tau}\right),
$$

where $y(\tau)$ is a modelled yield with given maturity $\tau$ and the parameter $\lambda$ specifies where loadings on curvature $c$ reach its peak. Fitting the yield curve separately every point in time $t$, we obtain series of coefficients $l_{t}, s_{t}$ and $c_{t}{ }^{2}$ We emphasise that the corresponding coefficients can be interpreted as level, slope and curvature. Hence, we have a dynamic factor model.

Just for illustration, the factor loadings for various $\tau$ and fixed $\lambda=0.0609$, which correspond to maturity $\tau=29.88$, where the curvature loadings attain its maximum, are presented in Figure 1. In this framework, the level factor $l_{t}$ loads equally on all maturities and can be directly interpreted in corresponding interest rates. The slope factor $s_{t}$ loads mostly on short-term maturities with exponentially decaying influence on longerterm rates. The coefficient $s_{t}$ represents slope and its rate of decay is determined by the coefficient $\lambda$. Lastly, the curvature factor $c_{t}$ loadings reach maximum in mid-term maturities, which is also determined by $\lambda$. Importantly, these factor loadings determine the shape of the designed yield curve shocks.

Figure 1

Factor Loadings

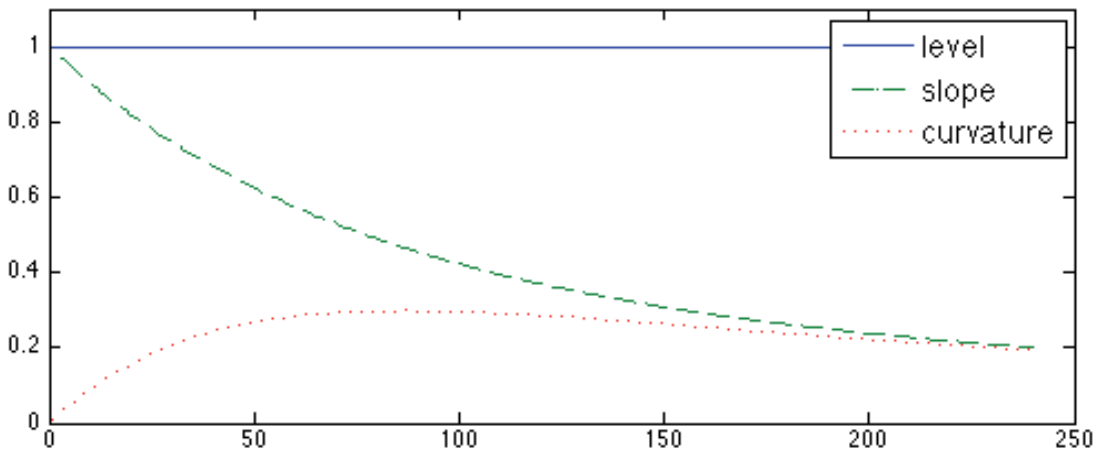

1 Over the time this model has become well-known; mainly as a yield curve smoothing tool (Diebold and $\mathrm{Li}, 2006$ ).

2 I.e. for a fixed $t$ we have an observed yield curve to which we can fit the Nelson-Siegel curve by a least squares estimator. 


\subsection{Dynamic Nelson-Siegel \& State Space Framework}

In this section, we present the dynamic Nelson-Siegel model specified and estimated using state space framework as presented in Diebold et al. (2006). This framework allows for clear statistical inference and produces correct standard errors, which can further be used for diagnostics and testing. An exhausting treatment regarding state space modelling can be found e.g. in Slavík (2005) and references therein.

The state space model constitutes of the state equation (2) and the observation equation (3). The former equation fits the yield curve and the latter specifies the dynamics of the coefficients of equation (1). Hence, we model the parameters of the Nelson-Siegel yield curve model as latent factors $l_{t}, s_{t}$ and $c_{t}$ for level, slope and curvature factor, respectively. Parameters $\mu_{l}, \mu_{s}, \mu_{c}$ represent the corresponding factor mean values and are modelled in efficient way as another latent factors with zero variance. As in Diebold et al. (2008), the latent factors follow vector autoregressive process of order 1 driven by transition matrix $T$.

$$
\left(\begin{array}{c}
l_{t+1}-\mu_{l} \\
s_{t+1}-\mu_{s} \\
c_{t+1}-\mu_{c}
\end{array}\right)=\underbrace{\left(\begin{array}{lll}
a_{11} & a_{12} & a_{13} \\
a_{21} & a_{22} & a_{23} \\
a_{31} & a_{32} & a_{33}
\end{array}\right)}_{T}\left(\begin{array}{c}
l_{t}-\mu_{l} \\
s_{t}-\mu_{s} \\
c_{t}-\mu_{c}
\end{array}\right)+\eta_{t}, \quad \eta \sim \mathrm{N}(0, Q)
$$

Although, we present the most general VAR(1) specification, we further estimate only diagonal elements of state covariance matrix $Q$ to keep the below shown regional model tractable. Furthermore, we denote a $p \times 1$ vector of yields of $p$ different maturities as $y_{t}=\left(\left(y_{t}\left(\tau_{1}\right), \ldots, y_{t}\left(\tau_{p}\right)\right)^{\prime}\right.$ and $p \times 3$ matrix of factor loadings as $Z$. The loadings are determined through equation (1) for all maturities.

$$
\left(\begin{array}{l}
y_{t}\left(\tau_{1}\right) \\
y_{t}\left(\tau_{1}\right) \\
\vdots
\end{array}\right)=\underbrace{\left(\begin{array}{cccc}
1 & \frac{1-e^{-\lambda \tau 1}}{\lambda \tau 1} & \frac{1-e^{-\lambda \tau 1}}{\lambda \tau 1} & -e^{-\lambda \tau 1} \\
1 & \frac{1-e^{-\lambda \tau 2}}{\lambda \tau 2} & \frac{1-e^{-\lambda \tau 2}}{\lambda \tau 2} & -e^{-\lambda \tau 2} \\
\vdots & \vdots & \vdots &
\end{array}\right)}_{Z}:\left(\begin{array}{l}
l_{t}-\mu_{l} \\
s_{t}-\mu_{s} \\
c_{t}-\mu_{c}
\end{array}\right)+\varepsilon_{t}, \varepsilon \sim \mathrm{N}(0, H),
$$

where $H$ is assumed to be diagonal, which implies uncorrelated disturbances in observation matrix. Diebold et al. (2006) stresses that the assumption of uncorrelated yields for different maturities is quite common. Moreover, it forces unobserved factors to capture the covariance between different maturities. The elements of the factor loadings matrix $Z$ are functions of maturity $\tau$ and parameter $\lambda$, which we fix to a mean value of series of monthly optima.

We estimated the model using Kalman filter, which delivers minimum mean square error estimates of each component in the state vector (latent factors $l_{t}, s_{t}$ and $c_{t}$ ) even if the observations are not normally distributed, given the linearity of estimators (Koopman et al., 1998, p. 67). The unknown parameters needed for the recursion are estimated using Maximum likelihood estimation as described in Durbin and Koopman 
(2001). In the most general case, we need to estimate $18+p$ parameters; 3 means in state equations, 9 elements in state transition matrix $T, 3$ diagonal elements and 3 off-diagonal elements in covariance matrix $Q$ and $p$ number of diagonal elements of in covariance matrix $H$ for $p$ number of maturities of yields. That means 30 parameters for CZK, HUF, PLN and SKK currency specific models.

\section{Regional Common Factor Model}

Before proceeding to specifying the novel regional model, we present principal component analysis on the stacked yield curves of the four countries which confirms that the four regional currencies are driven by common factors.

In Table 1, we show the results up to the fourth principal component. The first principal component (level) explains less variation of the data than in one currency only analysis. The second and the third component (slope and curvature, respectively) each explain over additional $10 \%$ of the variation in the data. Interestingly, the fourth principal component explains additional 5\% of the variation, which can still be considered. We presume that this component gathers some regional specifics not significant or observable on country level.

Table 1

Regional Principal Component Analysis

\begin{tabular}{|l|l|l|l|l|}
\hline & PC1 & PC2 & PC3 & PC4 \\
\hline Percentage & $68.03 \%$ & $11.89 \%$ & $10.99 \%$ & $5.65 \%$ \\
\hline Cumulative & $68.03 \%$ & $79.92 \%$ & $90.91 \%$ & $95.56 \%$ \\
\hline
\end{tabular}

Source: Authors' calculations

We base our further analysis and specification of the Regional Common Factor Model on two principal components. Therefore, we work with two strongest principal components known as the level and the slope components. These capture together near $80 \%$ of variation of the data. The paths of the two components are plotted in Figure 2.

Figure 2

\section{Regional Principal Components}

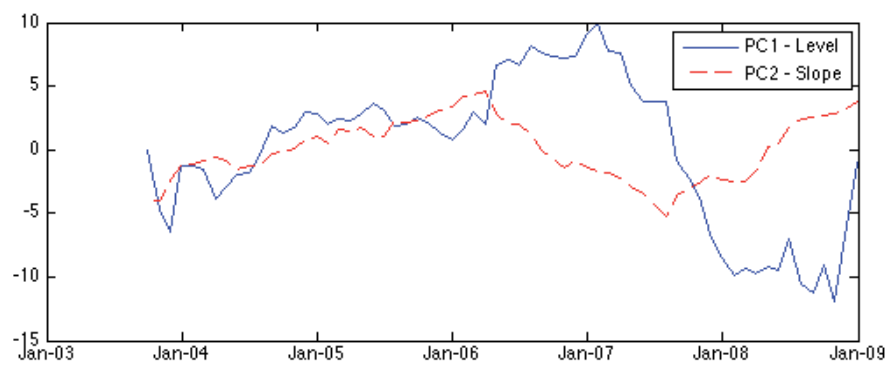

Further, we can build the Regional Common Factor Model, which is inspired by Diebold et al. (2008), yet some changes are introduced. Unlike single country models, the regional model combines several single currency models together to allow for extraction of regional common factors. 
We refer to our models as 'regional', because we use neighbouring currencies. Note that 'regional' yield curve is not observed, indeed, our approach does not need 'regional' yields, only country yields are used in the state space representation, see Equation (4). The model as such essentially combines Dynamic Nelson-Siegel models for all four countries of the Central European region and models them as one model, which provides a clear inference thus it can be estimated at once by Maximum likelihood estimator and the latent factors can be extracted by Kalman filter and smoother algorithms.

We begin by stacking the currency yield curves $y_{\text {currency, }, t\left(\tau_{i}\right)}$ (in one $(48 \times 1)$ column vector $Y_{t}\left(\tau_{i}\right)=\left(y_{C Z K, t,\left(\tau_{i}\right)}^{\prime}, \ldots, y_{S K K, t,\left(\tau_{i}\right)}^{\prime}\right)$. The crucial part is to correctly build the $(48 \times 12)$ loadings matrix $Z$ that translates the country specific factors and matrix $Z_{\text {regional }}$ that translates the regional factors into yields. The new loadings matrix contains original currencies' loadings matrices on the diagonal, which means that the regional latent factors load on the currencies' yield directly through matrix $Z_{\text {regional }}$. This specification estimates 14 latent factors, 12 summarised as $\alpha_{t}$ and the regional latent factors $L_{t}$ and $S_{t}$; for the implementation purposes, the model implicitly extracts 26 latent factors, since the factor means $\mu_{\text {currency } y}$ are modelled as latent factors as well in order to ease the estimation. Equation (4) presents the observation equation, which is obviously just an ordinary observation equation in state space formulation.

$$
Y_{t}(\tau)=\left(\begin{array}{cccc}
Z_{C Z K} & 0 & 0 & 0 \\
0 & Z_{H U F} & 0 & 0 \\
0 & 0 & Z_{P L N} & 0 \\
0 & 0 & 0 & Z_{S K K}
\end{array}\right) \alpha_{t}+Z_{\text {Regional }}\left(\begin{array}{l}
L_{t} \\
S_{t}
\end{array}\right)+\varepsilon_{t}, \varepsilon \sim \mathrm{N}(0, H),
$$

where $Z_{\text {regional }}$ denotes $(48 \times 2)$ loading matrix containing the scaled first two columns of $Z_{\text {currency }}$ as in equation (5); these two columns determine the loadings on the regional level and the regional slope latent factors.

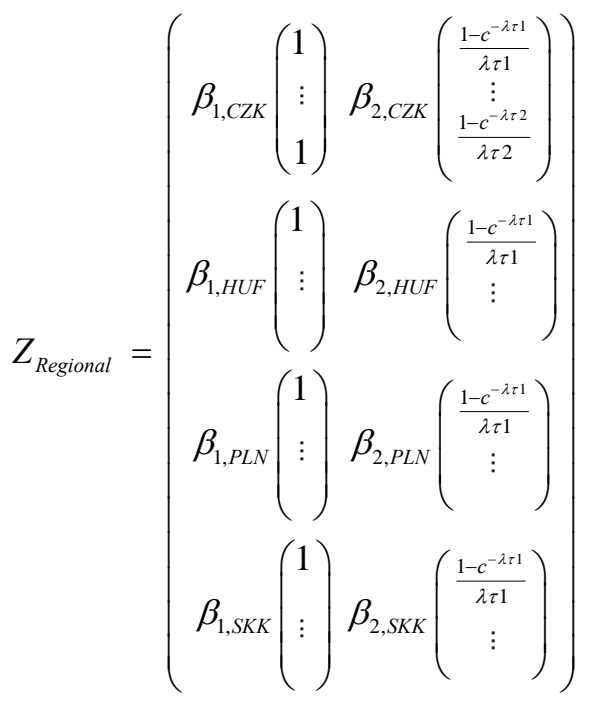


The coefficients $\beta$ represent the magnitude, which the currencies' yield curves load on the regional latent factors with. Since we not only put together four times the DNS model, somehow larger changes are made to the state matrix. In order to simplify the notation of the observation equation (4) and the state equation (9), we introduce a set of substitutions presented in equation (6); equation (7), which shows the structure of transition matrix $T$; and equation (8), which presents the regional latent factor transition matrix $\rho$.

$$
\alpha_{t}=\left(\begin{array}{c} 
\\
l_{C Z K, t}-\mu_{l, C Z K} \\
s_{C Z K, t}-\mu_{s, C Z K} \\
c_{C Z K, t}-\mu_{c, C Z K} \\
l_{H U F, t}-\mu_{l, H U F} \\
s_{H U F, t}-\mu_{s, H U F} \\
c_{H U F, t}-\mu_{c, H U F} \\
l_{P L N, t}-\mu_{l, P L N} \\
s_{P L N, t}-\mu_{s, P L N} \\
c_{P L N, t}-\mu_{c, P L N} \\
l_{S K K, t}-\mu_{l, S K K} \\
s_{S K K, t}-\mu_{s, S K K} \\
c_{S K K, t}-\mu_{c, S K K}
\end{array}\right)
$$

The simplifications in equation (7) that we estimate only the diagonal elements is rather technical and eases the optimisation of the log-likelihood function.

$$
T_{\text {currency }}\left(\begin{array}{ccc}
a_{11} & 0 & 0 \\
0 & a_{22} & 0 \\
0 & 0 & a_{33}
\end{array}\right)
$$

The state equation of this model needs to be adjusted in order to allow currency latent factors to load on regional factors. This is done by vectors $\beta_{\text {currency }}$, which enter the full model observation equation; equation (4) shows that we do not allow currency's level factor to load on regional slope factor and vice versa. There may be a natural relationship between the regional level and the country specific slope factors and vice versa, which we may miss.

The vectors $\beta_{\text {currency }}$ suggest that we do not assume any curvature factor to load on regional level or slope factors. The dynamics of the regional latent factor captures transition matrix $\rho$, in equation (8).

$$
\rho=\left(\begin{array}{ll}
\rho_{11} & \rho_{12} \\
\rho_{21} & \rho_{22}
\end{array}\right)
$$

The state equation (9) containing all the substitutions explains the currency factors' dynamics similarly to the Dynamic Nelson-Siegel model; as AR(1) processes. Furthermore, it also captures the regional factors' dynamics as vector AR(1) process, thus allowing for possible cross relations in regional factors. 


$$
\left(\begin{array}{c}
\alpha_{t+1} \\
L_{t+1} \\
S_{t+1}
\end{array}\right)=\left(\begin{array}{cccc|c}
T_{C Z E} & 0 & 0 & 0 & 0 \\
0 & T_{H U F} & 0 & 0 & 0 \\
0 & 0 & T_{P L N} & 0 & 0 \\
0 & 0 & 0 & T_{S K K} & 0 \\
\hline 0 & 0 & 0 & 0 & \rho
\end{array}\right)\left(\begin{array}{l}
\frac{\alpha_{t}}{L_{t}} \\
S_{t}
\end{array}\right)+\eta_{t}, \quad \eta \sim \mathrm{N}\left(0,\left(\begin{array}{cc}
Q & 0 \\
0 & I_{(2 \times 2)}
\end{array}\right)\right)
$$

We do not estimate the off-diagonal elements of state covariance matrix $Q$ to keep a reasonable complexity of the model. Note the structure of the matrix $T$ that in fact determines the whole dynamics of the model. Once again, in order to be able to identify the model, we introduce another restrictions on parameters. As in Diebold et al. (2008), we restrict $\beta_{l, C Z K}$ and $\beta_{2, C Z K}$ to be positive in order to have an identified model. We assume the regional factors' covariance matrix to be $(2 \times 2)$ unit matrix. The pleasant side effect is that the magnitude of coefficients $\beta$ can be directly interpreted as how much certain currency's factors load on regional factors.

Note that as in Diebold et al. (2008) the currencies' latent factors $l_{t}$ and $s_{t}$ represent currency specific idiosyncratic level and slope factors. Denoting $\eta_{l, t}$ and $\eta_{s, t}$ as currency specific level and slope latent factors corresponding to model with no explicitly modelled regional latent factors, we have the resulting equation (10) for a level factor of one of the currencies and in equation (11) for a slope factor of one of the currencies.

$$
\begin{array}{ll}
\eta_{l, t}=\mu_{l}+\beta_{1} L_{t}+l_{t}, & \operatorname{Var}\left(\eta_{l, t}\right)=\beta_{1}^{2} \operatorname{Var}\left(L_{t}\right)+\operatorname{Var}\left(l_{t}\right) \\
\eta_{s, t}=\mu_{s}+\beta_{2} L_{t}+s_{t}, & \operatorname{Var}\left(\eta_{s, t}\right)=\beta_{2}^{2} \operatorname{Var}\left(S_{t}\right)+\operatorname{Var}\left(s_{t}\right)
\end{array}
$$

Thus we model the currency specific latent factors through linear model with the regional latent factors acting as explanatory variables. Therefore, this yields the variance decomposition as specified in equation (10) and (11), which we use to assess influence of the regional factors on currencies' dynamics.

\section{Estimation and Results}

The estimation of such a complex model developed in this paper relies on methods for extracting signal from noisy measurements and mostly applied in engineering and physics. Kalman (1960) introduces a very efficient way of computing mean and variance of a noisy signal. Since the state space formulation has a state equation and an observation equation, it allows easy application of Kalman filter.

To ease the estimation, we introduce a number of restrictions on parameters and simplifications. While estimating the Regional Common Factor Model, we do not optimise the likelihood function over diagonal elements of matrix $H$, yet we use estimates of these values of separate currency specific models. As a result, we lowered the number of unknown parameters by $4 \times 12$. 


\subsection{Data}

Four different data-sets are used for estimating coefficients of our model based on zero rates. We obtained the zero rates from Thomson-Reuters for four Central European currencies: Czech koruna (CZK), Hungarian forint (HUF), Polish zloty (PLN) and Slovak koruna (SKK).

Due to limited data availability we restrict our analysis to shorter maturities, as the very long ones are not available with sufficient history and liquidity. The actual tenors for all four regional currencies are as following: $1 \mathrm{M}, 2 \mathrm{M}, 3 \mathrm{M}, 6 \mathrm{M}, 9 \mathrm{M}, 1 \mathrm{Y}, 3 \mathrm{Y}, 5 \mathrm{Y}$, $6 \mathrm{Y}, 7 \mathrm{Y}, 8 \mathrm{Y}$ and $10 \mathrm{Y}$. The sample length and number of maturities is shown in Table 2.

Table 2

Data-Set 2 Details

\begin{tabular}{|l|c|c|c|c|}
\hline Currency & Start Date & End Date & Tenors $p$ & Sample Size $n$ \\
\hline CZK & 31-Mar-1999 & 31-Nov-2009 & 12 & 117 \\
\hline HUF & 31-Mar-2002 & 31-Nov-2009 & 12 & 93 \\
\hline PLN & 31-Oct-2000 & 31-Nov-2009 & 12 & 110 \\
\hline SKK & 30-Sep-2003 & 12-Dec-2008 & 12 & 64 \\
\hline
\end{tabular}

Source: Thomson - Reuters

Looking at Figure 3, we can see some similarities in the evolution of countries' yield curves over time. We have to bear in mind different lengths of data sample and scale, e.g. parameters for the SKK model are further estimated using lower number of observations, since the SKK sample ends in December 2008. At first sight, the Czech koruna yield curve seems to be well behaved and is similar to those of strong currencies. If the history of the data allows, we observe periods of high rates around the year 2000, which were especially high in Polish zloty reaching over 15\%. In Figure 4, the Hungarian forint yield curve shows relatively high short-end volatility as well as high short-term rates, which is a sign that forint and Hungarian economy are vulnerable to temporary shocks.

The same figure shows many of common types of yield curve; upward sloping in case of CZK, downward sloping in case of HUF and almost flat in case of PLN and SKK.

Figure 3

\section{Regional Zero Yield Curves - Evolution over Time}
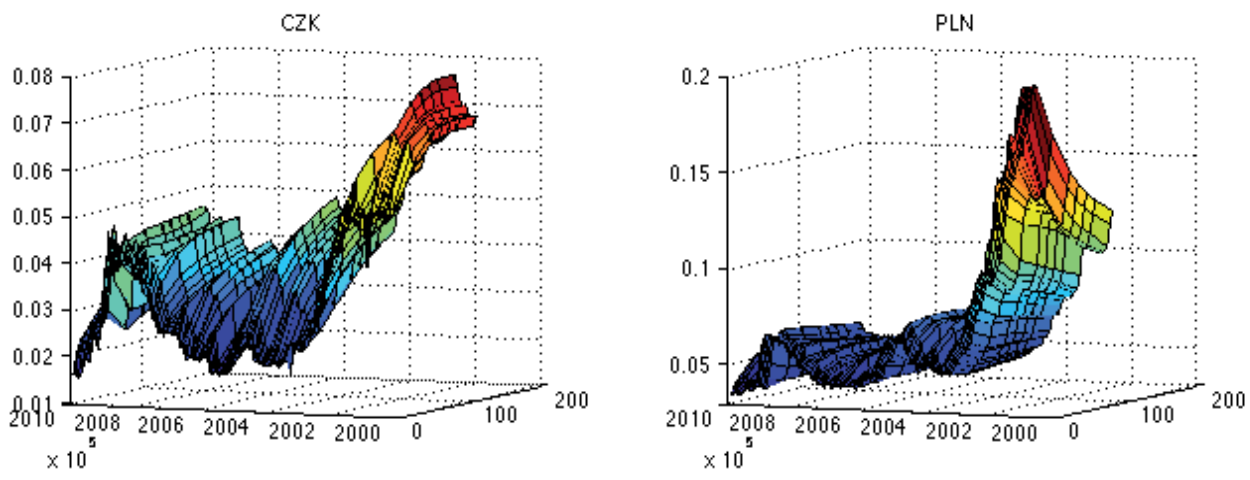
HUF

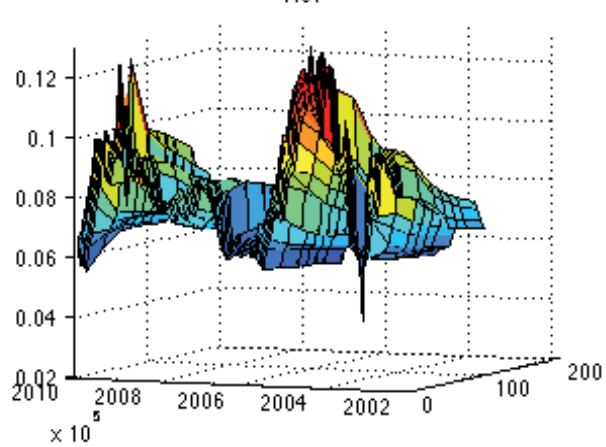

SKK

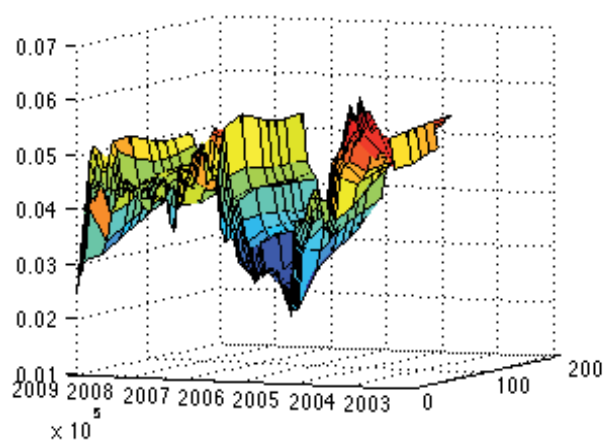

$\mathrm{x}$-axis denotes maturities $\tau$ in months; $z$-axis denotes interest rates

Source: Thomson - Reuters

Figure 4

Regional Zero Yield Curves - Empirical Quartiles
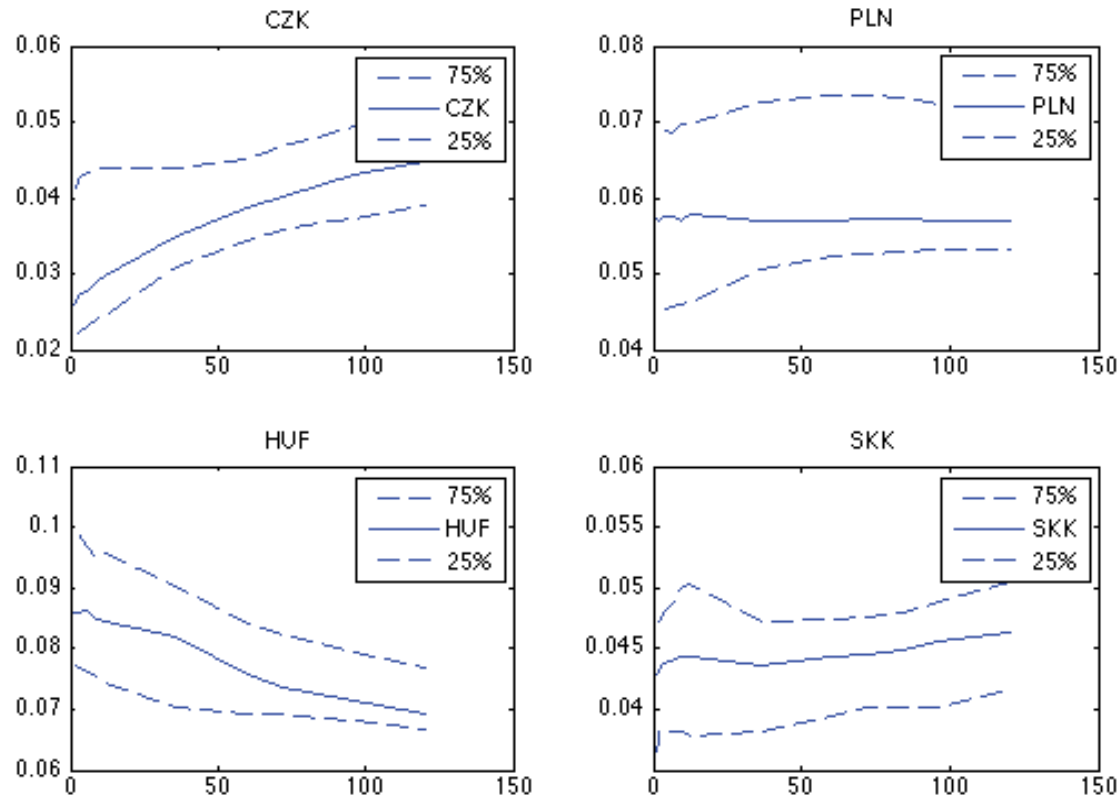

$x$-axis denotes maturities $\tau$ in months; $y$-axis denotes interest rates; ; 75\% / 25\% denoted by a dashed line represents the upper / lower quartile for a given maturity

Source: Thomson - Reuters 


\subsection{Regional Common Factor Model - Results}

This section proceeds with the estimation of the regional model. The latent factors are extracted with Kalman filter and the model parameters are estimated with Maximum likelihood estimator. ${ }^{3}$

This data-set includes the four regional currency yield curves. We adjusted the sample length to fit the shortest sub-sample available, which is the SKK sample starting 30-Sep-2003 and ending 31-Dec-2008, when the Slovak Republic accepted the common currency euro. Hence, we work with sample of $n=64$ observation and $p=48$ maturities. Hence, the sample has 3072 data-entries, and we estimate 36 parameters and extract 26 latent factors ${ }^{4}$.

The resulting estimates of elements of transition matrix $T$ and corresponding standard errors are in Table 3. For reader's convenience we put the diagonal elements into columns. The estimated means of currency specific factors and the estimates of currency loadings on regional factors are presented in Table 4.

Table 3

Estimates of Diagonal Elements of Transition Matrix $T$

\begin{tabular}{|l|c|c|c|c|c|c|c|c|}
\hline & \multicolumn{2}{|c|}{ CZK } & \multicolumn{2}{c|}{ HUF } & \multicolumn{2}{c|}{ PLN } & \multicolumn{2}{c|}{ SKK } \\
\hline coeff. & est. & SE & est. & SE & est. & SE & est. & SE \\
\hline $\mathbf{a}_{11}$ & 1.005 & 0.007 & 1.003 & 0.007 & 0.992 & 0.009 & 0.993 & 0.082 \\
\hline $\mathbf{a}_{22}$ & $0.945^{\star}$ & 0.027 & 0.919 & 0.066 & 0.980 & 0.044 & $0.846^{*}$ & 0.075 \\
\hline $\mathbf{a}_{33}$ & 0.892 & 0.067 & 0.857 & 0.081 & 0.950 & 0.046 & $0.871^{*}$ & 0.064 \\
\hline
\end{tabular}

* denotes element significantly different from 1 at $5 \%$ significance level.

Source: Authors' calculations

As we can see in Table 3, when removing the off-diagonal elements from currency sub-models and introducing the regional common factor for level and slope, all diagonal elements increased. ${ }^{5}$ Most of the diagonal elements is not significantly different from 1, which suggest two things: the model can be estimated with restricting the diagonal elements to 1 , which would mean assuming that the factors follow a random walk, and the regional common factors capture the 'predictable' dynamics.

According to results in Table 3, the only exceptions are the slope AR(1) coefficient in the CZK sub-model and the slope and the curvature AR(1) coefficient in the SKK sub-model. In Table 4, we see that the Czech koruna does not load on the regional slope factor almost at all, which keeps the currency slope AR(1) dynamics in place. In case of the SKK sub-model we see rather low slope loading, which possibly still keeps the $\mathrm{AR}(1)$ process for currency specific slope factor.

3 The estimation routine was implemented using Ox, an object-oriented matrix programming language - Ox version 5.00 (Doornik, 2007), in combination with SsfPack 2.2, a specialised package for estimation of state space models (Koopman, 1998).

$42 \times 12$ diagonal elements and variances of transition matrix $T+4$ elements of factor transition matrix $\rho+8$ coefficients $\beta=36$ parameters; 12 currency specific latent factors +12 currency specific factors means modelled as latent factor with 0 variance +2 regional factors $=26$ latent factors.

5 In comparison to model without the regional common factors. The estimated coefficients of currency specific models are available upon demand. 
Table 4

Estimates of Means $\mu$ and Vectors $\beta$

\begin{tabular}{|l|c|c|c|c|c|c|c|c|}
\hline & \multicolumn{2}{|c|}{ CZK } & \multicolumn{2}{c|}{ HUF } & \multicolumn{2}{c|}{ PLN } & \multicolumn{2}{c|}{ SKK } \\
\hline & est. & SE & est. & SE & est. & SE & est. & SE \\
\hline $\boldsymbol{\mu}_{1}$ & $-0.686^{\star}$ & 0.042 & $-1.789^{\star}$ & 0.497 & $-1.767^{*}$ & 0.496 & $-1.435^{\star}$ & 0.483 \\
\hline $\boldsymbol{\mu}_{\mathrm{s}}$ & $2.035^{\star}$ & 0.175 & $-0.301^{*}$ & 0.413 & $1.476^{*}$ & 0.370 & $0.759^{*}$ & 0.046 \\
\hline $\boldsymbol{\mu}_{\mathrm{c}}$ & $2.577^{\star}$ & 0.321 & $-0.160^{\star}$ & 0.405 & $1.937^{*}$ & 0.453 & $2.166^{\star}$ & 0.256 \\
\hline $\boldsymbol{\beta}_{1}$ & 0.022 & 0.141 & $0.266^{*}$ & 0.046 & $0.326^{*}$ & 0.052 & $0.197^{*}$ & 0.036 \\
\hline $\boldsymbol{\beta}_{2}$ & 0.003 & 1.195 & $0.421^{*}$ & 0.184 & 0.098 & 0.051 & 0.059 & 1.048 \\
\hline
\end{tabular}

* denotes element significantly different from 0 at $5 \%$ significance level.

Source: Authors' calculations

Since the Czech koruna does not load on the regional slope factor almost at all and because the principal component analysis ${ }^{6}$ revealed important slope component, we conclude there are country specific slope driving forces. The CZK sub-model also has the lowest coefficient $\beta_{1}$.

Although lower than the PLN $\beta_{l}$ coefficient, the HUF sub-model loads relatively a lot on the regional level factor compared to the CZK sub-model. The SKK sub-model follows a similar pattern to the CZK model, with higher loadings on regional level factor and low insignificant $\beta_{2}$ coefficient.

Table 5

Regional Latent Factor Transition Matrix

$$
\hat{\rho}=\left(\begin{array}{cc}
0.911 & 0.022 \\
(0.112) & (0.074) \\
0.535 & 0.695 \\
(0.306) & (0.140)
\end{array}\right)
$$

Table 6

Estimates of Diagonal Elements of $Q$

\begin{tabular}{|c|c|c|c|}
\hline$\hat{Q}_{\text {cZK }}$ & $\hat{Q}_{\text {HUF }}$ & $\hat{Q}_{\text {PLN }}$ & $\hat{Q}_{\text {SKK }}$ \\
\hline 0.042 & 0.057 & 0.052 & 0.030 \\
\hline 0.052 & 0.138 & 0.154 & 0.143 \\
\hline 0.667 & 3.710 & 0.786 & 0.849 \\
\hline
\end{tabular}

Source: Authors' calculations

The extracted regional factors are plotted in Figure 5. Table 6 shows the estimated variances of currency specific factors associated with currency sub-models. Generally

6 See appendix for details. 
speaking, by introducing two regional common factors the currency specific factors' variance decreased and the variation in currencies' yield is than explained by common factors.

To answer how much a specific currency's yields load on the common regional factors, we decompose the variance of the level or the slope currency specific latent factor into currency's idiosyncratic factor and the level or the slope regional factor, respectively, as in equation (10) and in equation (11). The results are in Table 7.

We see that the CZK latent factors possess its own dynamic, as the regional common factors explain very low percentage of the variance, $7.18 \%$ and $4.83 \%$ for the regional level and the regional slope factor respectively. Interestingly, the regional level factor explains up to $51.75 \%, 65.45 \%$ and $75.48 \%$ of the HUF, the PLN and the SKK level factor variance, respectively. This result seem reasonable as inflation - main determinant of the level factor - was lower and more stable in the Czech Republic than in other Visegrad countries for given period (Mehl, 2009). ${ }^{7}$

In contrary to the level factor, the PLN yield curve slope behaves differently than the regional slope factor. Mehl (2009) finds that the Polish yield curve slope is mainly linked to the USD yield curve slope. Also, the SKK slope factor is explained by the regional slope factor by less than $1 \%$. As the slope factor reflects major developments in the real economic activity, the results indicate that countries of our interest are quite heterogeneous with respect to their business cycle. This confirms partly also analysis based on the correlation coefficients of economic activity presented by CNB (2009, p. 30).

To complete the analysis, we plotted the extracted latent factors for each currency zone in model with and without the regional common factors in Figure 6. We see that the introduction of the common regional factors absorbed some of the variations in the data; e.g. the HUF slope factor is nicely smoothed out, whereas the CZK latent factors stayed almost the same.

Table 7

Variance Decomposition

\begin{tabular}{|l|l|c|c|c|}
\hline & CZK & HUF & PLN & SKK \\
\hline Level & $7.18 \%$ & $51.75 \%$ & $65.45 \%$ & $75.48 \%$ \\
\hline Slope & $4.83 \%$ & $78.93 \%$ & $1.86 \%$ & $0.48 \%$ \\
\hline
\end{tabular}

Source: Authors' calculations

7 Average y-o-y HICP in 2003-2009 for selected countries (\%): CZ: 2.3, HU: 5.3, SK: 4.1, PL: 2.8, CNB (2010), p. 61. 
Figure 5

Regional Common Factors

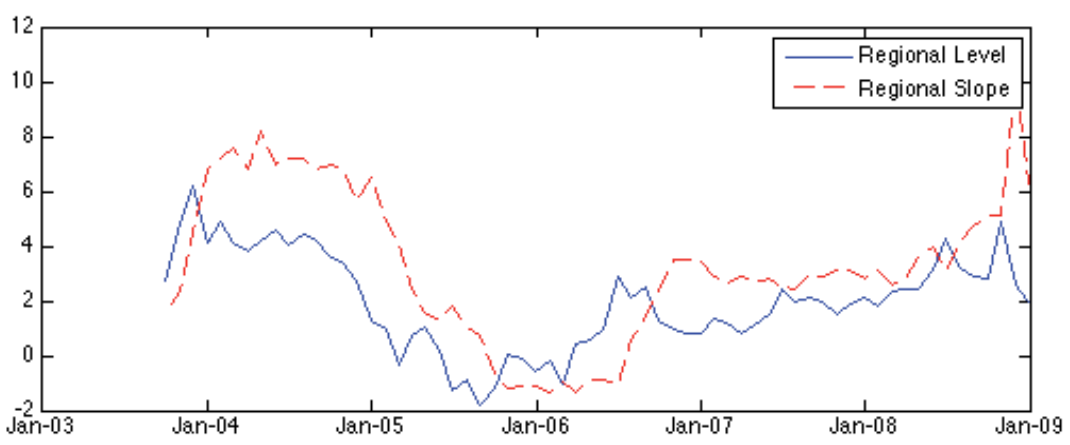

Figure 6

Currency Specific Latent Factors
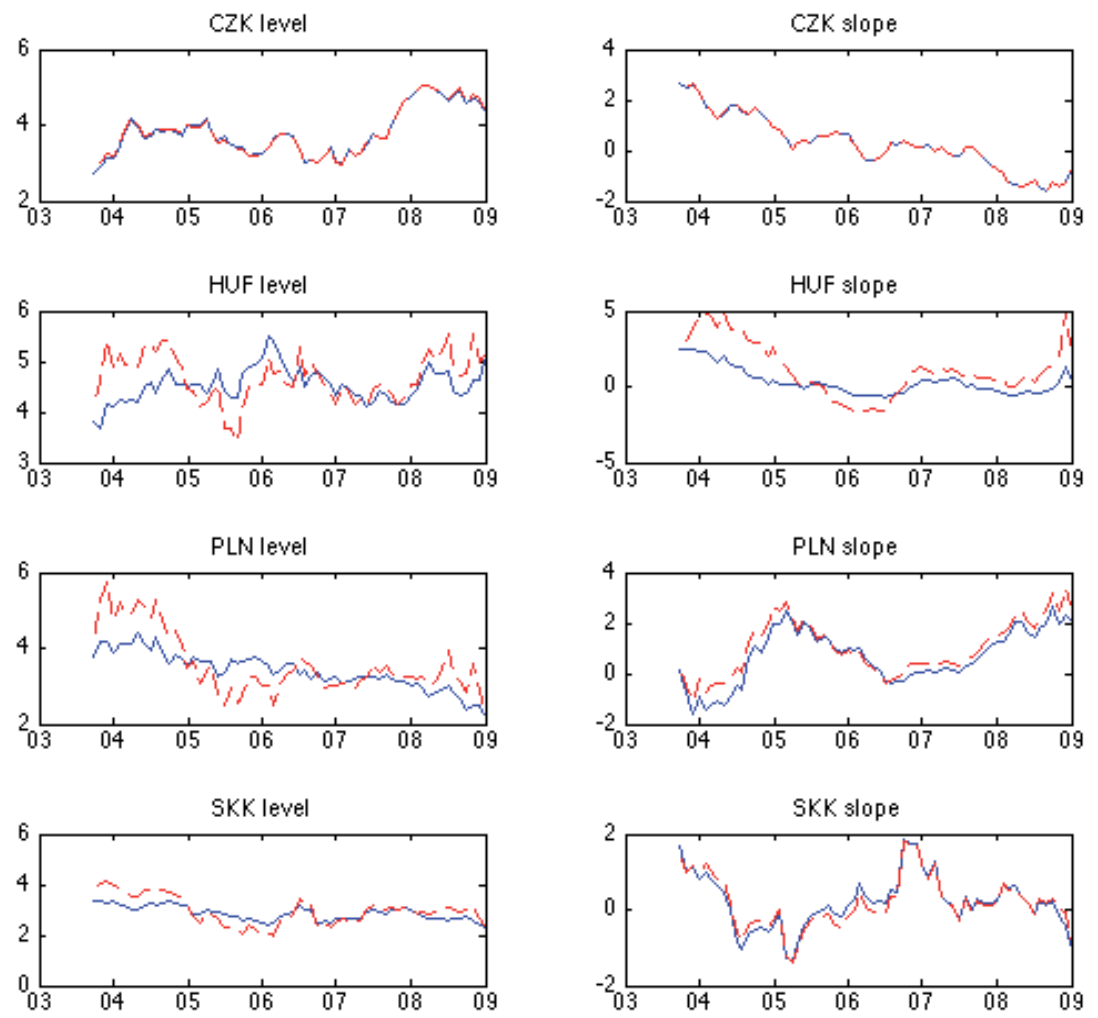

The solid line denotes extracted latent factors (level or slope) by the model with regional common factors, whereas the dashed line denotes the currency latent factors constructed in equation (10) and in equation (11). 


\section{Conclusion}

In this paper, we focus on and analyse dynamics of yield curves of various currency zones. We set off to modify dynamic Nelson-Siegel approach to capture driving factors behind regional yield curves and by using state space framework, we endeavour to extract regional common factors.

Furthermore, we propose a regional model based on global model by Diebold et al. (2008) who discovered global yield curve by analysing zero rates of USD, GBP, DM and JPY. Hence, we expected to find similar common dynamics in currencies' yield curves geographically even closer: CZK, HUF, PLN and SKK. In contrast to Diebold et al. (2008), we kept the currency specific curvature factor in the model to absorb the remaining variations in the data.

We model the regional yield curve as stacked currencies' yield curves. The proposed regional model relies on Kalman filter to extract the regional common factors, thus we refer to it as to the Regional Common Factor Model.

We found that almost all diagonal coefficients of transition matrices representing the main dynamics of latent factors seem to be quite persistent with values close to 1 . This suggests that possible random walk specification for the latent factors may be considered thus opening a new topic for further research.

The regional model brought information on how much currencies' sub-models load on regional factors and how much of the variation in the yield curve data of each currency is explained by the regional common factors. We find that the HUF yield curve loads the most on both regional factors and these loading coefficients are significant, whereas the CZK level factor loads the least on the regional factors. Additionally, we confirmed that zloty's slope factor behave to a large extent independently of regional slope factor, which Mehl (2009) explains by stronger link to the USD yield curve. Moreover, the SKK yield curve dynamics does not load on the regional slope factor at all, yet the regional level factor explains over $75 \%$ of the variance of the SKK level factor. Due to low loadings and low percentage of factor variation assigned to the regional factors, we conclude that the CZK yield curve possesses its own dynamics corresponding to country specific features. 


\section{Appendix}

To accompany our analysis, we performed additional principal component analysis on each currency's yield curve separately. This analysis confirms findings by Litterman and Scheinkman (1991) and identifies three main components responsible for the variation in the data. The results are in Table 8 . We see that dynamics of each currency's yield curve is strongly driven by the first principal component followed by the much weaker second and minor third component.

Table 8

Currency Specific Principal Component Analysis

\begin{tabular}{|c|c|c|c|c|}
\hline & & $\begin{array}{c}\text { PC1 } \\
\text { Level }\end{array}$ & $\begin{array}{l}\text { PC2 } \\
\text { Slope }\end{array}$ & $\begin{array}{c}\text { PC3 } \\
\text { Curvature }\end{array}$ \\
\hline \multirow{2}{*}{ N } & Percentage & $92.93 \%$ & $6.33 \%$ & $0.54 \%$ \\
\hline & Cumulative & $92.93 \%$ & $99.25 \%$ & $99.80 \%$ \\
\hline \multirow{2}{*}{ 로 } & Percentage & $92.98 \%$ & $5.09 \%$ & $1.38 \%$ \\
\hline & Cumulative & $92.98 \%$ & $98.07 \%$ & $99.45 \%$ \\
\hline \multirow{2}{*}{$\frac{z}{a}$} & Percentage & $98.37 \%$ & $1.45 \%$ & $0.15 \%$ \\
\hline & Cumulative & $98.37 \%$ & $99.82 \%$ & $99.98 \%$ \\
\hline \multirow{2}{*}{$\frac{r}{\mathfrak{r}}$} & Percentage & $91.24 \%$ & $5.14 \%$ & $2.77 \%$ \\
\hline & Cumulative & $91.24 \%$ & $96.38 \%$ & $99.15 \%$ \\
\hline
\end{tabular}

Source: Authors' calculations 


\section{References}

CNB (2009), Analyses of the Czech Republic's Current Economic Alignment with the Euro Area. Prague: Czech National Bank, December 2009.

CNB (2010), Inflation Report II/2010. Prague: Czech National Bank, May 2010.

Diebold, F. X., Li, C. (2006), "Forecasting the Term Structure of Government Bond Yields." Journal of Econometrics, 130, pp. 337-364.

Diebold, F. X., Rudebusch, G. D., Aruoba, B. (2006), "The Macroeconomy and the Yield Curve: A Dynamic Latent Factor Approach." Journal of Econometrics, 131, pp. 309-338.

Diebold, F. X., Ji, L., Li, C. (2006), "A Three-Factor Yield Curve Model: Non-Affine Structure, Systematic Risk Sources, and Generalized Duration," in Klein, L. R. (ed.), Long-Run Growth and Short-Run Stabilization: Essays in Memory of Albert Ando. Cheltenham, U.K.: Edward Elgar, pp. 240-274.

Diebold, F. X., Li, C., Yue, V. (2008), "Global Yield Curve Dynamics and Interactions: A Generalized Nelson-Siegel Approach." Journal of Econometrics, 146, pp. 351-363.

Doornik, J. A. (2007), Object-Oriented Matrix Programming Using Ox. 3rd ed. London: Timberlake Consultants Press.

Durbin, J., Koopman, S. J. (2001), Time Series Analysis by State Space Methods. Oxford University Press, 2001, ISBN 978-0-19-852354-3.

Hoffmaister, A. W., Roldós, J., Tuladhar, A. (2010), "Yield Curve Dynamics and Spillovers in Central and Eastern European Countries." IMF Working Paper, WP/10/51, 2010.

Kalman, R. E. (1960), "A New Approach to Linear Filtering and Prediction Problems. Transactions of the ASMA." Journal of Basic Engineering, Series D, 82, pp. 35-45.

Koopman, S. J., Shephard, N., Doornik, J. A. (1998), "Statistical Algorithms for Models in State Space Using SsfPack 2.2." Econometrics Journal, Vol. 1, pp. 1-55.

Litterman, R., Scheinkman, J. (1991), "Common Factors Affecting Bond Returns." Journal of Fixed Income, 1, pp. 54-61.

Nelson, C. R., Siegel, A. F. (1987), "Parsimonious Modelling of Yield Curve." Journal of Business, 60, pp. 473-489.

Málek, J., Radová, J., Štěrba, F. (2007), "Yield Curve Construction Using Government Bonds in the Czech Republic." Politická ekonomie, 2007, 55(6), pp. 792-808.

Marciniak, M. (2006), "Yield Curve Estimation at the National Bank of Poland: Spline Based Methods, Curve Smoothing and Market Dynamics." Bank i kredyt, No. 10.

Mehl, A. J. (2009), "The Yield Curve as Predictor and Emerging Economies." Open Economies Review, 20(5), pp. 683-716.

Reppa, Z. (2009), "A Joint Macroeconomic-Yield Curve Model for Hungary.” Central Bank of Hungary Working Papers, 1/2009.

Slavík, M. (2005), "Introduction to Time Series Modeling: State Space Models and Kalman Filter." Politická ekonomie, 2005, 53(1), pp. 111-124.

Svensson, L. E. O. (1995), "Estimating Forward Interest Rates with the Extended Nelson-Siegel Method." Quarterly Review, No. 3, pp. 13-26, Sveriges Riksbank. 